\title{
Production d'impulsions de photons dans I'UV-VUV
}

\author{
E. Robert, C. Cachoncinlle, A. Khacef et J.M. Pouvesle \\ GREMI, CNRS, Université d'Orléans, BP. 6759, 45067 Orléans cedex 02, France
}

\begin{abstract}
Résumé: Nous présentons une synthèse des fluorescences produites dans nos conditions expérimentales, dans le domaine UV-VUV $(120-400 \mathrm{~nm})$, lors de l'excitation par rayonnement $\mathrm{X}$ et par décharge à barrière diélectrique de milieux à base de gaz rares à des pressions (multi)atmosphériques. Dans ce travail, les longueurs d'ondes des émissions, l'origine des fluorescences et les conditions d'excitation sont répertoriés pour les espèces atomiques ou moléculaires, neutres ou ioniques.
\end{abstract}

\section{INTRODUCTION}

Dans le cadre des recherches sur les sources de photons énergétiques, les gaz rares présentent un intérêt évident de part leur configuration électronique. Cependant leur excitation et/ou ionisation requiert la mise en oeuvre de techniques de "pompage » énergétiques. Dans cette optique, nous avons développé deux méthodes de production de photons basées sur l'emploi de décharges impulsionnelles H.T.: les décharges à barrière diélectrique (DBD) et les Flashes X. Les impulsions d'excitation, X ou électriques, déclenchées permettent une spectroscopie nanoseconde résolue en temps à l'aide de tubes photomultiplicateurs et oscilloscope rapides. Nous présentons ici une synthèse des fluorescences, produites dans nos conditions d'excitation, dans le domaine UV-VUV. Certaines fluorescences sont rapportées ici pour la première fois.

\section{RESULTATS}

\subsection{Longueurs d'ondes issues d'états neutres atomiques ou moléculaires}

L'excitation (par DBD ou X) des gaz rares purs conduit, à basse pression, à l'émission des raies de résonance $\left(\mathrm{Kr}^{*}: 116 \mathrm{~nm}, \mathrm{Xe}^{*}: 147 \mathrm{~nm}\right)$ puis, à plus haute pression, à l'émission des premiers et seconds continus $\left(\mathrm{Ar}_{2}{ }^{*}: 126 \mathrm{~nm}, \mathrm{Kr}_{2}{ }^{*}: 146 \mathrm{~nm}, \mathrm{Xe}_{2}{ }^{*}: 178 \mathrm{~nm}\right)$. Par transfert d'excitation à partir d'états $\mathrm{Ar}^{*}$ nous observons les transitions du radical hydroxyl $\left(\mathrm{OH}^{*}: 306 \mathrm{~nm}\right)$ et du second système positif de $\mathrm{N}_{2}\left(\mathrm{~N}_{2}{ }^{*}: 297\right.$, $316,337,357$ et $380 \mathrm{~nm}$ ) [1]. En DBD, l'excitation de mélanges à base de gaz rares a permis de mettre en évidence les fluorescences identifiées par Johnson [2] de; $\operatorname{ArO}^{*}: 186$ et $203 \mathrm{~nm}, \mathrm{XeO}^{*}: 234 \mathrm{~nm}$, de $\mathrm{KrCl}^{*}: 222 \mathrm{~nm}$, comme présenté sur la figure 1, ainsi que le continu $\mathrm{H}_{2}{ }^{*}$ enregistré dans nos conditions entre $180 \mathrm{~nm}$ et $300 \mathrm{~nm}$ [3]. Dans le cas d'une excitation par DBD le mélange gazeux se trouve enrichi par des atomes du matériau constituant les parois diélectriques de la cellule comme le chlore et l'oxygène. En excitation par flash X nous avons observé l'émission $\left(\mathrm{H}^{*}: 121 \mathrm{~nm}\right)$ [4] dans des mélanges $\mathrm{Ne} / \mathrm{H}_{2}$.

\subsection{Longueurs d'ondes issues d'états ioniques moléculaires}

Nos études ont permis l'identification des continus moléculaires ioniques de l'argon et du krypton. Nous avons montré que ces émissions sont dues aux dimères et aux trimères ioniques des gaz rares une fois et/ou deux fois chargés. Les longueurs d'ondes de ces continus se situent, en excitation par flash $X$, à: 
$\left(\mathrm{Ar}_{\mathbf{n}}{ }^{\mathrm{m+*}}: 160,185,200,225\right.$ et $245 \mathrm{~nm}, \mathrm{Kr}_{\mathrm{n}}{ }^{\mathrm{m+*}}: 170,225,230,270$ et $\left.300 \mathrm{~nm}, \mathrm{Xe}_{\mathbf{n}}{ }^{\mathrm{m}+*}: 170-500 \mathrm{~nm}\right)$ [5]. En $\mathrm{DBD}$, le continu très large bande du Ne a été mesuré entre $180 \mathrm{~nm}$ et $500 \mathrm{~nm}$ [6]. L'excitation par flash X des mélanges binaires de gaz rares conduit à la fluorescence des ions hétéronucléaires de gaz rares respectivement: (ArHe ${ }^{+}: 143$ et $146 \mathrm{~nm}$ [4], $\mathrm{ArNe}^{+}: 216$ et $224 \mathrm{~nm}[1], \mathrm{Ar}^{+} \mathrm{Xe}: 328$ et $508 \mathrm{~nm}$ [1]).

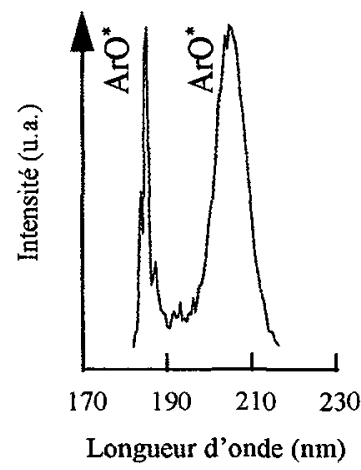

a)

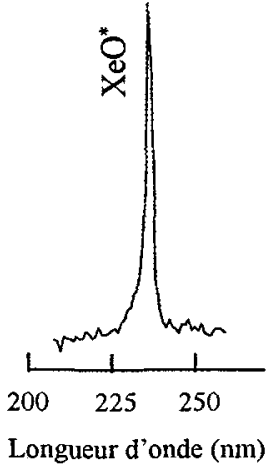

b)

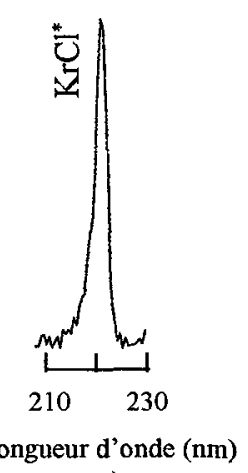

c)

Figure 1: Spectres en excitation par DBD des mélanges a) $\mathrm{Ar}$ ( 1250 torr $) / \mathrm{O}_{2}$ ( 6 torr), b) $\mathrm{Ar}(800$ torr $) / \mathrm{Xe}$ (0.2 torr)/O(impureté) et c) $\mathrm{Kr}$ ( 800 torr)/Cl(impureté).

\section{CONCLUSIONS}

Les DBD appliquées à des gaz rares ou des mélanges à base de gaz rares se révèlent être des sources intéressantes de rayonnement UV-VUV. Ces sources sont mises à profit dans divers procédés technologiques dont des dispositifs destinés à l'amélioration de l'environnement [7] (dépollution, valorisation...). Le flash $\mathrm{X}$ offre pour sa part des possibilités assez uniques en tant que source d'excitation et d'études de fluorescence. De plus, il permet de mener des études cinétiques dans des plasmas à haute pression entre autre dans des mélanges à base de gaz rares et dans des milieux favorables au développement de nouvelles sources, éventuellement cohérentes, dans l'UV-VUV.

\section{Remerciements}

Ce travail a bénéficié du soutien financier du programme INTAS 96-0351 et de la Société de Secours des Amis des Sciences.

\section{Références}

[1] E. Robert et al., XXIII ${ }^{\text {rd }}$ ICPIG, III, 32 (1997).

[2] D.E. Johnson, Chem. Phys. Letters, 238, 71 (1995).

[3] C. Cachoncinlle, thèse Université d'Orléans (1991).

[4] L. Huré et al., $8^{\text {th }}$ Int. Symposium on Light Sources, edited by G. Babucke, 300 (1998).

[5] E. Robert et al., Opt. Comm., 117, 179 (1995).

[6] J.M. Pouvesle et al., Spectral Line Shapes, 7, 359 (1993).

[7] C. Hibert et al., ce volume (1998). 